\title{
Journal of Bone and Mineral Metabolism Best Paper Award 2019
}

() The Japanese Society Bone and Mineral Research and Springer Japan KK, part of Springer Nature 2019

The Journal of Bone and Mineral Metabolism Best Paper Award was established in 2008. Candidates for the award must be members of the Japanese Society of Bone and Mineral Research, and the winner is honored at the Society's Annual Meeting.

We are pleased to announce that the following article has received JBMM Best Paper Award.

"The effects of switching daily teriparatide to oral bisphosphonates or denosumab in patients with primary osteoporosis"

by

Kosuke Ebina, Jun Hashimoto, Masafumi Kashii,Makoto Hirao, Shoichi Kaneshiro, Takaaki Noguchi, Yasunori Tsukamoto, Hideki Yoshikawa

J Bone Miner Metab (2017) 35:91-98

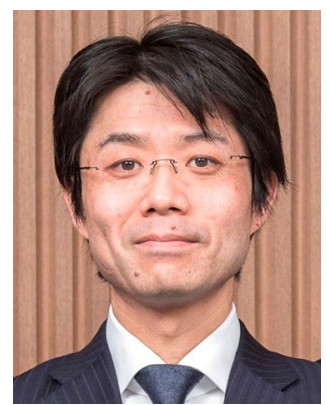

Dr. Kosuke Ebina

\begin{abstract}
The aim of this 12-month, observational study was to compare the effects of switching daily teriparatide (TPTD) to oral bisphosphonates (BP) therapy or denosumab (DMAb) therapy in patients with primary osteoporosis. Patients $[n=78 ; 71$ postmenopausal women and seven men; mean age 76.3 (64-94) years; mean duration of prior daily TPTD therapy 20.1 (6-24) months] were allocated to either the (1) "switch-to-BP" group [ $n=36$; weekly alendronate $35 \mathrm{mg}(n=$ 19), weekly risedronate $17.5 \mathrm{mg}(n=12)$, monthly minodronate $50 \mathrm{mg}(n=5)]$; or (2) "switch-toDMAb" group ( $n=42 ; 60 \mathrm{mg}$ sc every 6 months) based on each physicians' decision. Changes in bone mineral density (BMD) and serum bone turnover markers were monitored every 6 months. No significant difference was observed in baseline clinical characteristics between the groups. After 12 months, the increase in BMD was significantly greater in the switch-to-DMAb group compared to the switch-to-BP group: lumbar spine (6.2 vs. $2.6 \% ; P<0.01)$, total hip (4.2 vs. $1.1 \% ; P<0.05$ ), and femoral neck (3.5 vs. $1.4 \% ; P<0.05)$. In addition, the patients in the switch-to-DMAb group showed a significant decrease compared to those in the switch-to-BP group in TRACP-5b $(-55.8$ vs. $-32.8 \% ; P<0.01)$ and ucOC ( -85.5 vs. $-65.0 \% ; P<0.001)$, while no significant difference was observed in PINP (-67.5 vs. $-62.1 \%$ ). Switching daily TPTD to DMAb significantly increased BMD and decreased bone resorption marker compared to switching to oral BP at 12 months, and thus may provide an effective sequential treatment option after daily TPTD treatment.
\end{abstract}

We offer our sincere congratulations on behalf of the Journal of Bone and Mineral Metabolism, with best wishes for further development of the author's research.

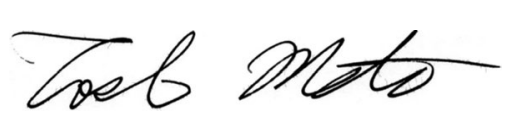

Toshio Matsumoto

Editor-in-Chief

Journal of Bone and Mineral Metabolism

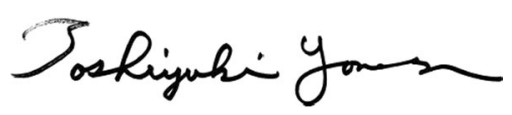

Toshiyuki Yoneda

Editor-in-Chief

Journal of Bone and Mineral Metabolism 